\title{
Genetics and oncology (part 1.) Fundamentals of genetic testing-based personalised medicine in oncology
}

\author{
Maria M. Sąsiadek , Izabela Łaczmańska1,2, Adam Maciejczyk²,3, Rafał Matkowski2,3, Justyna Gil
}

\author{
${ }^{1}$ Department of Genetics, Wroclaw Medical University, Wroclaw, Poland \\ ${ }^{2}$ Wroctaw Comprehensive Cancer Center, Wroclaw, Poland \\ ${ }^{3}$ Department of Oncology, Wrocław Medical University, Wroclaw, Poland
}

The dynamic development of genetics in recent decades has opened a new era in medicine. Understanding molecular mechanisms of multiple human diseases has laid the foundations for targeted medical care, based on knowledge of the basic pathogenesis of these diseases. This breakthrough is particularly evident in oncology because knowledge of the molecular basis of cancer leads to a change in the paradigm of medical care for the patients. Gradually, classification and treatment based only on organ location and histopathologic diagnosis is becoming outdated, and so is the classification considering clinical stage and malignancy of the tumour. Personalized treatment for individual patients based on the profile of genetic changes is increasingly common. Defining the genetic aetiology of neoplastic diseases was an achievement that allowed for division of neoplasms into sporadic ones and those which develop due to hereditary predisposition. It also enabled establishment of the molecular classification of neoplasms and more and more frequently - targeted treatment and precise clinical prognosis.

This article is the first one in a series of articles written by oncologists and geneticists. We hope that this series will be helpful for oncologists in understanding genetic problems and for geneticists - in understanding oncologic issues.

Key words: personalised medicine, oncology, molecular classification, predictive tests, prognostic tests

\section{Introduction}

Personalized (targeted, precise) medicine is based on knowledge of the genetic aetiology of a disease, and its objective is to introduce medical treatment adapted to specific molecular alterations that cause pathology [1-3]. The underlying concept of this approach is to optimise therapy by using precisely targeted drugs, and thus minimizing side effects and optimising patient care costs, as targeted therapy reduces the risk of administration of a therapy which is ineffective or leads to adverse effects [1-3].

Thus defined, the idea of personalized medicine is not new - it was authored by Paul Erlich (Nobel prize laureate in
1908), who developed the concept of causal treatment (magic bullet) based on identification of the pathogenic agent [4].

In oncology, introduction of personalised procedures into clinical practice has become possible with development of testing techniques that allow identification of genetic changes and molecular pathways that are key in the aetiology of cancer, and which are present or absent in individual patients with the same histopathological diagnosis of the tumour [1].

Currently, mainly genomic and genetic testing techniques are applied to identify the "molecular target of personalised medicine" (mutations of individual genes, chromosome aberrations, methylation disorders). These techniques

\section{How to cite:}

Sąsiadek MM, Łaczmańska I, Maciejczyk A, Matowski R, Gil J. Genetics and oncology (part 1.). Fundamentals of genetic testing-based personalised medicine in oncology. NOWOTWORY J Oncol 2020; 70: 144-149. 
include next generation sequencing (NGS), cytogenic and molecular cytogenetic tests: multiplex ligation-dependent probe amplification (MLPA), fluorescent in situ hybridization (FISH), array comparative genomic hybridization (aCGH), gene expression studies, and soon, proteomic testing techniques will be available, too $[5,6]$.

\section{Genetic basis of cancer}

In research aimed at understanding the genetic background of cancers, a breakthrough achievement occurred in early 1970s with development of a model of inheriting retinoblastoma based on the analysis of incidence of this neoplasm in affected families, and with development of the theory concerning mechanism of suppressor genes' effect in this model (Knudson's two hit hypothesis) [7, 8]. Publications by Knudson and al. encouraged research concerning neoplasms and nowadays, after years of epidemiologic analyses, family clinical studies and along with genetic research, 5-10\% of neoplasms are known to belong to hereditary cancer syndromes, most commonly characterised by autosomal dominant, and more rarely - autosomal recessive inheritance $[9,10]$.

About $15 \%$ of cancer cases are familial and are determined by multifactorial inheritance mechanism (interaction of environmental, potentially carcinogenic and genetic factors that increase individual sensitivity to their effects), and the remaining approximately 75\% develop as a sporadic disease [11].

Studies of the constitutional genome of cancer patients and cancer cells have led to the conclusion that they are two different genomes: the constitutional shows stability and invariability throughout lifetime, while cancer cell genome are highly heterogeneous and unstable. The instability of the latter genome is the reason for its variability both in an individual patient in the course of tumour progression, as well as in different cancer patients with the same histopathological diagnosis [12].

Genetic studies have shown that cancers classified in a single group based on histopathological studies represent actually many different types. This may be evidenced by lung cancer, traditionally classified as small-cell cancer and non-small cell cancer (adenocarcinoma and squamous cell cancer). However, genetic tests of lung cancer cells have revealed a vast complexity of its molecular forms [13].

Tumour development is a process stretched over time (most commonly it lasts 5-20 years), and its multi-stage course is determined by mutations which accumulate in the cell, leading to a change in its biological properties [14]. In 2011, Hanahan and Weinberg defined eight major biological features of cancer and two potential ones:

- proliferation independent from signals stimulating cell division,

- no reaction to proliferation inhibitors,

- no programmed cell death,

- replication immortality,
- angiogenesis,

- activation of infiltration and metastasis processes,

- genome instability,

- inflammation that promotes neoplasia,

- changes in energy metabolism,

- "escape" from the immune system's "supervision" mechanism. [15].

Currently, researchers consider two major theories of neoplastic transformation as grounded: clonal and cancer stem cells theory. Both refer to expansion of cancer cells, development of genetically variable cell clones and selection of clones of the highest potential for proliferation and adaptation to the tissue eco-system $[16,17]$. The difference between these theories lies in different properties of the first cell from which the transformation process begins. According to the clonal theory, the transformation is triggered by a random cell in the body where the first mutation occurs; while according to the cancer stem cell theory - by a cancer stem cell. Cancer stem cells form a small (below 1\%) subpopulation of tumour cells of particular biological properties, e.g. low proliferation potential, no capacity of final differentiation, presence of characteristic surface markers [17].

In the neoplastic transformation, the main role is played by three groups of genes: oncogenes, suppressor genes and mutator genes. Oncogenes are activated forms of protooncogenes which are present in every cell of the body. In the process of neoplastic transformation, they stimulate cell proliferation. For most cancers, it is possible to identify an oncogene which is the leading genetic force (driver mutation) responsible for uncontrolled cell division. This phenomenon is called oncogene addiction. Suppressor genes are classified as "gatekeepers" because they control cell division points, directing mutated cells to a path of repair of DNA damage or to programmed death (apoptosis). Finally, mutator genes are referred to as "caretakers" and they are responsible for removing unpaired and mispaired bases from DNA, as such bases are the cause of mutation [14].

Cancer development, clinical course and response to therapy are affected by these three groups of genes of key impact on neoplastic transformation (genes of high penetrance), but also by many genes of moderate and low penetrance (e.g. genes which are involved in the process of angiogenesis, cell array adhesion, affect the organism's immune reaction, localised tumour development, metastasis potential, reaction to therapy and many other processes) [14].

In the process of neoplastic transformation all those genes are interconnected in complex networks of mutual interdependence. Thus, they are all regulated by other genes located upstream on the signalling pathway (upstream genes), and they themselves regulate activity of downstream genes. This is the "vertical" regulation system, and at the same time mutual interrelations of genes are expressed in "horizontal" bonds - e.g. through modification of the tumour ecosystem 
on the local (tissue) level and in the entire body (e.g. immune reactions) [18].

Functional alterations of oncogenes, suppressor genes and mutator genes lead to genetic instability of cancer cells. Instability may be expressed on the chromosome level (aberrations of the number and structure of chromosomes), gene level (accumulation of mutations) or in alterations of epigenetic regulation of gene expression ( global hypomethylation which contributes to cells' chromosomal instability and hypermethylation of suppressor and mutator genes, thus leading to loss of their function). Accumulation of genetic alterations in cells causes changes of their biological properties and also leads to development of resistance to the therapy $[14,19]$.

\section{Personalized care for cancer patients}

Personalised medicine in oncology should be offered to patients with inherited cancer syndromes, as well as patients with sporadic neoplasms.

Carriers of critical mutations which determine inheritance of this syndrome receive personalised medical care including:

1. Prophylaxis: for most hereditary cancer syndromes, the increased risk concerns not only a specific, individual organ, but also other ones within the risk spectrum. This can be illustrated by the hereditary non-polyposis colorectal cancer (HNPCC), also referred to as Lynch syndrome. Its spectrum includes colonic cancer, but also cancers of the endometrium, ovary, bile ducts, urinary tract, stomach and brain [20]. Knowledge of this spectrum allows optimisation of prophylaxis by planning a test program or resection of healthy organs from the spectrum (depending on the risk of developing cancer).

2. Chemoprevention (prophylactic drug administration aimed at reduction of the risk of cancer development, e.g. administration of tamoxifen) in carriers of BRCA1/BRCA2 gene mutation [21].

3. Personalisation of medical management, e.g. special recommendations concerning surgical management in carriers of BRCA1 and BRCA2 mutations and concerning targeted management (e.g. olaparib in patients with ovarian cancer and hereditary and/or somatic mutation of BRCA1/BRCA2) [21].

4. Genetic counselling for patients and their families, provided by clinical geneticists and based on the analysis of pedigree and clinical data and results of genetic tests. With a family and clinical analysis it is possible to diagnose or suspect the hereditary cancer syndrome and to determine the scope of genetic testing for the individual patient, but it also enables interpretation of the genetic test results in the clinical context. Moreover, it is possible to identify family members, who may carry the critical mutation, and further to select the optimal method of genetic testing for those people and genetic counselling with information on the risk of further transfer of the mutation [22]. Predictive tests, i.e. those performed in healthy people with a family risk of cancer, are legally allowed in adults and should always be performed on two independent material samples.

\section{Rules for selection of genetic diagnosis methods in patients with cancer for the purpose of personalised medicine}

Regardless of whether neoplasm development is due to inherited, family, or sporadic factors, cancer cells have their own genome of specific properties described above.

If molecular changes are identified, it is possible to determine the following markers:

- diagnostic - supporting the diagnosis process,

- predictive - enabling forecasting of response to the applied treatment,

- prognostic - allowing determination of prognosis. For this purpose, testing of DNA isolated from cancer cells is needed.

Choosing the right method of genetic testing is crucial, both for medical and economic reasons.

\section{Genetic diagnosis in hereditary cancer syndromes}

The objective of genetic testing is to identify hereditary mutations, and the tested material is isolated DNA from somatic cells (usually lymphocytes of peripheral blood, as well as skin fibroblasts or mucosal cells - smear of the internal aspect of cheek).

Most of hereditary cancer syndromes are characterized by high genetic diversity, despite the same clinical manifestations of the disease. This phenomenon is described by the concepts of genetic, allelic, and non-allelic heterogeneity. The term allelic heterogeneity means that there is more than one mutation in a critical gene (e.g., about 1,200 mutations are known in the BRCA1 gene). Meanwhile, non-allelic heterogeneity occurs when the same disease may be conditioned by pathogenic variants in different genes. One example of non-allelic heterogeneity is the hereditary breast/ovarian cancer syndrome, which may be conditioned by mutations in multiple genes, however the most common mutations occur in BRCA1 gene (approximately $25 \%$ of patients with this syndrome) and BRCA2 (another 25\% of patients), and in the remaining group of patients the syndrome may occur due to mutation of such genes as: ATM, BARD1, BLM, BRIP1, CHEK2, MLH1, MRE11, MSH2, MSH6, NBN, PALB2, PMS2, PTEN, RAD51C, RAD51D, STK11, TP53 [23].

Out of these genes, some (e.g. BRCA1 and BRCA2) belong to the "high-penetrance genes" group, which means that carriers of their pathogenic variants have a defined, high risk of developing breast/ovarian cancer. Other genes in this group are classified as "moderate-penetrance genes", which moderately increase the risk of development of a cancer and the forecast risk is based on an analysis of a genetic test result and family history of cancer [24]. In the case of some genes, researchers 
have described a phenomenon of preferential occurrence of some mutations in a specific population - "founder mutations" (e.g. in the case of BRCA1 gene, in the Polish population about $50 \%$ of patients with mutation of this gene have one of the following three mutations: C.5266dupC (former name: 5382insC), c.4035delA (former name: 4153delA), c.181T > G p.Cys61Gly (former name: C61G) [23, 25].

The situation becomes even more complicated, if one considers that not all genetic changes have the same clinical consequences. The pathogenicity of some variants is known, and clinical management standards have been developed for their carriers. On the other hand, other variants are rarely described, their pathogenicity has not yet been clearly defined, while available knowledge and bioinformatic analysis allow to classify them as potentially pathogenic changes. Some changes have not been described so far and constitute the group of lesions of unknown clinical significance (variants of unknown significance - VUS). In the ClinVar database, among the 9,073 described variants of the BRCA1 and BRCA2 genes, approximately 2197 are variants with unknown pathogenicity [26]. As databases are constantly updated and new variants are constantly characterised, the result of NGS test analysis in a patient should indicate the date of accessing the databases and the obtained data should be stored for potential re-analysis in future.

In this complicated genetic situation, there is still no consensus concerning the scope of genetic tests to be recommended for patients with specific clinical problems.

Some authors claim that the optimal recommendation is to sequence all genes which potentially may be critical for the hereditary cancer syndrome in question (clinical panel). The benefits of this approach include reduced testing costs and reduced waiting time, as well as effective use of isolated DNA. There are also negative consequences: increased number of identified variants of unknown pathogenicity or variants in genes for which no standard clinical proceeding has been developed, as well as identification of changes in genes of moderate and low penetrance, leading to a difficult situation for the patient and doctor, when targeted clinical management cannot be introduced, even though the genetic change is known [27].

Other authors claim that the request should include testing only those genes for which there are clinical procedures developed (targetable mutations). Some countries (e.g. United Kingdom) have developed official diagnostic recommendations, e.g. Recommendations of the UK Cancer Genetics Group (2018) for diagnostics of genes whose mutations determine the risk of occurrence of hereditary breast (BRCA1, BRCA2, PALB2, PTEN, STK11, TP53) or ovarian (BRCA1, BRCA2, MLH1, MSH2, MSH6, PMS2, RAD51C, RAD51D) cancer syndromes [28].

\section{Personalized management based on genetic changes in cancer cells}

Identification of genetic changes in cancer cells allowed better understanding of molecular mechanisms of neoplastic trans- formation, and thus, it enabled more precise, cause-based classification of cancers and development of targeted treatment. Cancer cells may be tested using DNA isolated from the primary or metastatic tumour cells, or else from cancer cells or cancer DNA circulating in the patient's blood (liquid biopsy). Consistency of the genetic tests results for the primary tumour and lymph node metastases with those for other primary tumours is uncertain, while comparison of test results for primary tumours and material circulating in the patients' serum may give variable results [29].

The genetic heterogeneity of cancer tumours and the fact that tumours of the same histopathological diagnosis differ essentially in genetic aetiology explains to a large extent the variability of patients' responses to standardised therapies and different clinical course of the disease. Currently, a molecular classification is being developed for an increasing number of cancers that allows for precise biological differentiation of tumours. This is crucial for choosing personalized clinical management. This may be evidenced by the molecular classification of brain gliomas (WHO Classification, 2016), which allows identification of different forms of low-differentiated gliomas: distinguishing primary glioblastoma multiforme (without IDH1/IDH2 mutation, with presence of: $10 q$ deletion, PTEN mutation, EGFR amplification, CDKN2A/2B deletion) from secondary glioblastoma multiforme (with IDH1/IDH2 mutation) originating oligoastrocytomas (IDH1) IDH2 mutation, 1p/19q co-deletion, TP53 mutation and 9p deletion), diffuse astrocytomas (IDH1/IDH2 and TP53 mutations, 17p, 9p, 20q deletions) or anaplastic oligodendrog lioma (1p/19q co-deletion and deletion of 9p and 10q) [30]

As genetic testing of cells of various cancers is increasingly broadly applied, it was found that there are several common pathways of signal transmission which stimulate neoplastic transformation, e.g. the pathway starting from activation of the epidermal growth factor receptor (EGFR) or tyrosine kinase (RTK), which lead to stimulation of cell proliferation [31]. Understanding that the same signalling pathways may be activated in different neoplasms leads to a modification of rules of classification of neoplastic tumours for the purpose of targeted management: the molecular classification has become important parallelly to the organ-based classification. The increasing share of medication is applied molecularly. Consequently, patients with different cancers - but with the same mutations - are treated with the same drugs [32]. For example, there is a treatment which involves blocking of factors that stimulate hyperproliferation and it is the same for cancers that differ in location and histopathology, but "depend" on the same oncogene, such as application of:

- trastuzumab in breast and stomach cancers in which the key molecular change is amplification of the HER2 gene

- crozitinib in patients with non-small cell lung cancer with ALK mutations,

- gefitinib in tumours of the same histopathologic type, but with EGFR amplification, 
- imatinib in cancer patients with the KIT mutation or $B C R$ / ABL fusion gene,

- vemurafenib in cancer patients with BRAF mutations $[32,33]$.

Molecular testing of cancer cells allowed also for explanation of the phenomenon of non-identical response to targeted treatment in patients with the same leading molecular change, e.g. EGFR amplification. Different studies, e.g. concerning metastases of colonic cancer, have shown modification of functions of multiple genes involved in the signalling pathway in neoplasms, making downstream genes independent from genes located upstream the signalling path which normally regulate their expression (EGFR - RAS - BRAF - MEK / ERK or EGFR - PI3K-AKT and PTEN) [31, 32].

This complicated system of genetic relationships in cancer cells leads to further dilemmas in genetic diagnostics. There is a question whether assessment of prognostic markers before initiation of the targeted therapy should rely on individual genes which mutate the most commonly (e.g. EGFR amplification) or a panel of genes on the specific signalling pathway. There are no specific guidelines for management of most tumours yet, e.g. for metastatic colonic cancer. The US Food and Drug Administration (US FDA) has already approved a panel for testing KRAS and NRAS gene mutations to allow identification of 56 specific mutations in exons 2, 3 and 4 of these genes [34].

Treatment of cancer patients with drugs selected on the basis of molecular changes is a very promising trend in therapy. However, usually after approximately two years of treatment, patients acquire resistance to the therapy [31, 32].

The mechanisms of acquired resistance to treatment vary, but they can be classified in two main groups:

1. Internal tumour resistance (intrinsic resistance), which results from the high genetic instability of cancer cells and leads to a rapid change in their genetic characteristics, both spontaneous and in response to the treatment used (leading to the elimination of dominant cell clones, which are replaced by less numerous clones of different genetic characteristics).

2. Induced resistance (acquired resistance), which arises in response to treatment and results from:

- activation (through mutations) of genes located on the signal pathway below the gene which is the current "target (effector)" of treatment (activation of upstream effector),

- activation of another oncogene that stimulates cell proliferation (bypass, bypass of (onco) protein effector),

- activation of another signalling pathway (kinase target) [35].

\section{Prognostic and predictive tests}

The clinical and genetic heterogeneity of neoplastic diseases means that frequently it is not possible to precisely predict the course of neoplastic disease for an individual patient. This is a serious medical, psychological, and social problem. Therefore, for years researchers have been striving to develop molecular tests that would allow forecasting of different aspects of the disease, e.g. overall survival rate or survival rate before metastases. Despite many years of research and multiple predictive tests offered on the market, none of them has been approved for routine application in clinical practice yet.

Currently, many predictive tests are available, meant for patients with various neoplasms, but the highest number of tests is designed for patients with breast cancer. These tests are based on analysis of expression of various genes in the tumour tissue and they differ both in the scope of predictive potential and analysed genes.

The most commonly used tests include: Mammaprint, Oncotype Dx Breast, Prosigna PAM-50 Breast Cancer Prognostic Gene Signature Assay, Breast Cancer Index (BCI) and EndoPredict. All four tests are intended for patients post breast tumour resection, with known hormonal status and condition of lymph nodes, as well as size and grade of the tumour. These tests assess the risk of distant recurrence (and Oncotype DX also assesses the response to chemotherapy) [36-38].

Prostate cancer is another type of cancer that occurs frequently and displays great clinical variability. Patients with this disease have access to two main tests available on the market, which forecast course of the disease. These are Oncotype Dx Genomic Prostate Score and Genomic Classifier, Decipher (based on assessment of 22 RNA markers).

Currently, research is underway to develop prognostic tests for patients with other cancers, too: urinary bladder cancer (Decipher Bladder), cancers with unknown primary (Response Dx, CancerTYPE ID, Rosetta Cancer Origin, ProOnc, SourceDX, PathfinderTG), colonic cancer (Oncotype DX Colon Cancer Assay, Colorectal Cancer DSA, GeneFx Colon, OncoDefender (RC), leukaemia (FoundationOne ${ }^{\oplus}$ Heme) or melanoma (Decision DxMelanoma, Decision Dx-UM, DermTech PLA). However, it has not been proven yet, whether these tests are clinically relevant [37].

\section{Conclusion}

Development of personalised medicine in oncology leads to a change of the paradigm of understanding neoplasms, and thus also to a change of the broadly defined medical care for oncological patients and their families. Only cooperation between oncologists and geneticists will allow introduction of truly personalised medical care based on understanding of the genetic background of cancer.

Conflict of interest: none declared

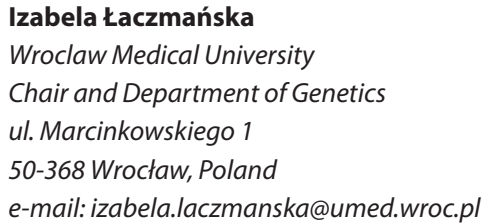




\section{References}

1. Filipp FV. Precision medicine driven by cancer systems biology. Cancer Metastasis Rev. 2017; 36(1): 91-108, doi: 10.1007/s10555-017-9662-4, indexed in Pubmed: 28265786.

2. Ginsburg GS, Phillips KA. Precision Medicine: From Science To Value. Health Aff (Millwood). 2018; 37(5): 694-701, doi: 10.1377/ hlthaff.2017.1624, indexed in Pubmed: 29733705.

3. Vogenberg FR, Isaacson Barash C, Pursel M. Personalized medicine: part 1: evolution and development into theranostics. PT. 2010; 35(10): 560-576, indexed in Pubmed: 21037908.

4. Strebhardt K, Ullrich A. Paul Ehrlich's magic bullet concept: 100 years of progress. Nat Rev Cancer. 2008; 8(6): 473-480, doi: 10.1038/nrc2394, indexed in Pubmed: 18469827.

5. Kowalik A, Kowalewska M, Góźdź S. Current approaches for avoiding the limitations of circulating tumor cells detection methods-implications for diagnosis and treatment of patients with solid tumors. Transl Res. 2017; 185: 58-84.e15, doi: 10.1016/j.trsl.2017.04.002, indexed in Pubmed: 28506696

6. Ribeiro IP, Melo JB, Carreira IM. Cytogenetics and Cytogenomics Evaluation in Cancer. Int J Mol Sci. 2019; 20(19), doi: 10.3390/ijms20194711, indexed in Pubmed: 31547595.

7. Knudson AG. Mutation and cancer: statistical study of retinoblastoma. Proc Natl Acad Sci U S A. 1971; 68(4): 820-823, doi: 10.1073/ pnas.68.4.820, indexed in Pubmed: 5279523.

8. Knudson AG, Hethcote HW, Brown BW. Mutation and childhood cancer: a probabilistic model for the incidence of retinoblastoma. Proc Natl Acad Sci U S A. 1975; 72(12): 5116-5120, doi: 10.1073/pnas.72.12.5116, indexed in Pubmed: 1061095.

9. Nagy R, Sweet K, Eng C. Highly penetrant hereditary cancer syndromes. Oncogene. 2004; 23(38): 6445-6470, doi: 10.1038/sj.onc.1207714, indexed in Pubmed: 15322516.

10. Garber JE, Offit K. Hereditary cancer predisposition syndromes. J Clin Oncol. 2005; 23(2): 276-292, doi: 10.1200/JCO.2005.10.042, indexed in Pubmed: 15637391.

11. McCartan $D$, Chatterjee $S$. Hereditary and familial cancer. Surgery (Oxford). 2018; 36(3): 145-150, doi: 10.1016/j.mpsur.2017.12.003.

12. Stratton MR, Campbell PJ, Futreal PA. The cancer genome. Nature. 2009; 458(7239): 719-724, doi: 10.1038/nature07943, indexed in Pubmed: 19360079.

13. Kutkowska J, Porębska I, Rapak A. Non-small cell lung cancer - mutations, targeted and combination therapy. Postepy Hig Med Dosw (Online). 2017; 71(0): 431-445, doi: 10.5604/01.3001.0010.3826, indexed in Pubmed: 28513466.

14. Bashyam MD, Animireddy $S$, Bala $P$, et al. The Yin and Yang of cancer genes. Gene. 2019; 704: 121-133, doi: 10.1016/j.gene.2019.04.025, indexed in Pubmed: 30980945.

15. Hanahan D, Weinberg RA. Hallmarks of cancer: the next generation. Cell. 2011; 144(5): 646-674, doi: 10.1016/j.cell.2011.02.013, indexed in Pubmed: 21376230.

16. Greaves M, Maley CC. Clonal evolution in cancer. Nature. 2012; 481(7381): 306-313, doi: 10.1038/nature10762, indexed in Pubmed: 22258609.

17. Gil J, Stembalska A, Pesz KA, et al. Cancer stem cells: the theory and perspectives in cancer therapy. J Appl Genet. 2008; 49(2): 193-199, doi: 10.1007/BF03195612, indexed in Pubmed: 18436993.

18. Fronczak A. Medycyna personalizowana. Mity, fakty, rekomendacje. Plexus s.c, Łódź 2016.

19. Vargas-Rondón N, Villegas VE, Rondón-Lagos M. The Role of Chromosomal Instability in Cancer and Therapeutic Responses. Cancers (Basel). 2017; 10(1), doi: 10.3390/cancers10010004, indexed in Pubmed: 29283387.

20. Møller P. The Prospective Lynch Syndrome Database reports enable evidence-based personal precision health care. Hered Cancer Clin
Pract. 2020; 18: 6, doi: 10.1186/s13053-020-0138-0, indexed in Pubmed: 32190163.

21. King MC, Wieand S, Hale K, et al. National Surgical Adjuvant Breast and Bowel Project. Tamoxifen and breast cancer incidence among women with inherited mutations in BRCA1 and BRCA2: National Surgical Adjuvant Breast and Bowel Project (NSABP-P1) Breast Cancer Prevention Trial. JAMA. 2001; 286(18): 2251-2256, doi: 10.1001/jama.286.18.2251, indexed in Pubmed: 11710890.

22. Lewis KM. Identifying hereditary cancer: genetic counseling and cancer risk assessment. Curr Probl Cancer. 2014; 38(6): 216-225, doi: 10.1016/j. currproblcancer.2014.10.002, indexed in Pubmed: 25432528.

23. McClellan J, King MC. Genetic heterogeneity in human disease. Cell. 2010; 141(2): 210-217, doi: 10.1016/j.cell.2010.03.032, indexed in Pubmed: 20403315.

24. Kiwerska K, Szyfter K. DNA repair in cancer initiation, progression, and therapy-a double-edged sword. J Appl Genet. 2019; 60(3-4): 329-334, doi: 10.1007/s13353-019-00516-9, indexed in Pubmed: 31468363.

25. Kowalik A, Siołek M, Kopczyński J, et al. BRCA1 founder mutations and beyond in the Polish population: A single-institution BRCA1/2 next-generation sequencing study. PLoS One. 2018; 13(7): e0201086, doi: 10.1371/journal.pone.0201086, indexed in Pubmed: 30040829.

26. Landrum MJ, Lee JM, Benson M, et al. ClinVar: improving access to variant interpretations and supporting evidence. Nucleic Acids Res. 2018; 46(D1): D1062-D1067, doi: 10.1093/nar/gkx1153, indexed in Pubmed: 29165669.

27. Gagan J, Van Allen EM. Next-generation sequencing to guide cancer therapy. Genome Med. 2015; 7(1): 80, doi: 10.1186/s13073-015-0203-x, indexed in Pubmed: 26221189.

28. Taylor A, Brady AF, Frayling IM, et al. UK Cancer Genetics Group (UKCGG). Consensus for genes to be included on cancer panel tests offered by UK genetics services: guidelines of the UK Cancer Genetics Group. J Med Genet. 2018; 55(6): 372-377, doi: 10.1136/jmedgenet-2017-105188, indexed in Pubmed: 29661970.

29. Qiu J, Xu J, Zhang K, et al. Refining Cancer Management Using Integrated Liquid Biopsy. Theranostics. 2020; 10(5): 2374-2384, doi: 10.7150/thno.40677, indexed in Pubmed: 32089746.

30. Louis DN, Perry A, Reifenberger G, et al. The 2016 World Health Organization Classification of Tumors of the Central Nervous System: a summary. Acta Neuropathol. 2016; 131(6): 803-820, doi: 10.1007/ s00401-016-1545-1, indexed in Pubmed: 27157931.

31. Yamaoka T, Kusumoto S, Ando K, et al. Receptor Tyrosine KinaseTargeted Cancer Therapy. Int J Mol Sci. 2018; 19(11), doi: 10.3390/ ijms19113491, indexed in Pubmed: 30404198.

32. Krzyszczyk P, Acevedo A, DavidoffEJ, et al. The growing role of precision and personalized medicine for cancer treatment. Technology (Singap World Sci). 2018; 6(3-4): 79-100, doi: 10.1142/S2339547818300020, indexed in Pubmed: 30713991.

33. Helgadottir H, Rocha Trocoli Drakensjö I, Girnita A. Personalized Medicine in Malignant Melanoma:Towards Patient Tailored Treatment. Front Oncol. 2018; 8: 202, doi: 10.3389/fonc.2018.00202, indexed in Pubmed: 29946532.

34. Afrăsânie VA, Marinca MV, Alexa-Stratulat T, et al. KRAS, NRAS, BRAF, HER2 and microsatellite instability in metastatic colorectal cancer - practical implications for the clinician. Radiol Oncol. 2019; 53(3): 265-274, doi: 10.2478/raon-2019-0033, indexed in Pubmed: 31553708.

35. von Manstein V, Yang CM, Richter D, et al. Resistance of Cancer Cells to Targeted Therapies Through the Activation of Compensating Signaling Loops. Curr Signal Transduct Ther. 2013; 8(3): 193-202, doi: 10.2174/15 74362409666140206221931 , indexed in Pubmed: 25045345.

36. Smith A, Farrah K. Gene Expression Profiling Tests for Breast Cancer: A Rapid Qualitative Review. Canadian Agency for Drugs and Technologies in Health. ; 2019.

37. Vieira AF, Schmitt F. An Update on Breast Cancer Multigene Prognostic Tests-Emergent Clinical Biomarkers. Front Med (Lausanne). 2018; 5: 248, doi: 10.3389/fmed.2018.00248, indexed in Pubmed: 30234119.

38. Schildgen V, Warm M, Brockmann M, et al. Oncotype DX Breast Cancer recurrence score resists inter-assay reproducibility with RT-Profiler Multiplex RT-PCR. Sci Rep. 2019; 9(1): 20266, doi: 10.1038/s41598-01956910-0, indexed in Pubmed: 31889145. 